\title{
HIV AND STI BEHAVIOURAL SURVEILLANCE AMONG MEN WHO HAVE SEX WITH MEN IN EUROPE
}

\author{
J Elford (j.elford@city.ac.uk)1, A Jeannin², B Spencer², J P Gervasoni², M J van de Laar³, F Dubois-Arber², the HIV and STI \\ Behavioural Surveillance Mapping Group ${ }^{4}$
}

1. City University, London, United Kingdom

2. Institute for Social and Preventive Medicine (IUMSP), University of Lausanne

3. European Centre for Disease Prevention and Control (ECDC), Stockholm, Sweden

4. Members of the group are listed at the end of the article

This article was published on 26 November 2009.

Citation style for this article: Elford J, Jeannin A, Spencer B, Gervasoni JP, van de Laar MJ, Dubois-Arber F, the HIV and STI Behavioural Surveillance Mapping Group. HIV and STI behavioural surveillance among men who have sex with men in Europe. Euro Surveill. 2009;14(47):pii=19414. Available online: http://www.eurosurveillance.org/ ViewArticle.aspx?ArticleId $=19414$

\begin{abstract}
This paper describes behavioural surveillance for HIV and sexually transmitted infections (STI) among men who have sex with men (MSM) in Europe, focusing on the methods and indicators used. In August 2008, questionnaires were sent to European Union Member States and European Free Trade Association countries seeking information on behavioural surveillance activities among eight population groups including MSM. Thirty-one countries were invited to take part in the survey and 27 returned a questionnaire on MSM. Of these 27 countries, 14 reported that there was a system of behavioural surveillance among MSM in their country while another four countries had conducted behavioural surveys of some kind in this subpopulation. In the absence of a sampling frame, all European countries used convenience samples for behavioural surveillance among MSM. Most European countries used the Internet for recruiting and surveying MSM for behavioural surveillance reflecting increasing use of the Internet by MSM for meeting sexual partners. While there was a general consensus about the main behavioural indicators (unprotected anal intercourse, condom use, number of partners, HIV testing), there was considerable diversity between countries in the specific indicators used. We suggest that European countries reach an agreement on a core set of indicators. In addition we recommend that the process of harmonising HIV and STI behavioural surveillance among MSM in Europe continues.
\end{abstract}

\section{Introduction}

In many European countries men who have sex with men (MSM) are at increased risk for HIV and other sexually transmitted infections (STI) such as syphilis or gonorrhoea. Even though there has been an increase in the number of new HIV diagnoses among heterosexual men and women in some European countries in recent years, MSM still remain at greatest risk of acquiring HIV in Europe [1]. As a consequence, a number of European countries have conducted behavioural surveys among MSM to monitor HIV and STI risk in this population [2]. In some countries these surveys are part of a well established behavioural surveillance programme while in others they are conducted on an ad hoc basis.

Behavioural surveillance allows us to monitor, at a population level, risks related to HIV and STI transmission. Trends in risk behaviour can provide a valuable insight into corresponding trends in disease incidence over time. In addition, behavioural surveillance provides information for planning and evaluating prevention interventions.

In 2008, a study was conducted to map HIV and STI behavioural surveillance activities in European Union (EU) Member States and European Free Trade Association (EFTA) countries, focusing on the methods and indicators used for behavioural surveillance. Information was collected on behavioural surveillance in eight different populations, specifically the general population, young people, MSM, injecting drug users (IDU), people living with HIV or AIDS (PLWHA), sex workers, people attending STI clinics, migrants and ethnic minorities. In this paper we describe the key findings from the mapping exercise concerning behavioural surveillance among MSM in Europe.

\section{Methods}

In 2008, questionnaires were sent to all EU Member States and EFTA countries concerning behavioural surveillance activities in each country (see Table 1 for list of countries). Each country received nine separate questionnaires. One questionnaire concerned national behavioural surveillance and second generation surveillance systems. The remaining eight questionnaires addressed each of the specific subpopulations. It was emphasised on each questionnaire that the focus was behavioural, as opposed to biological surveillance. Behavioural surveillance was defined as "the collection and use of data from different sources and/or different time points to globally ascertain the state and evolution of the HIV/AIDS and/or STI epidemics at the behavioural, as opposed to biological, level".

In the population-specific questionnaires we asked whether a behavioural surveillance system was currently in place for that population. If so, each country was asked to provide information about their methodology for conducting behavioural surveillance in that population. In particular, they were asked to provide information on the year(s) when they had conducted behavioural surveys since 1985 in that group, the sampling method, data collection and the main topics covered. The main topics were grouped under the following headings: knowledge and attitudes, sexual relationships and sexual partners, sexual activity and lifestyle, exposure to risk of infection, HIV and STI, drugs and substance use. Information was 
also requested on the main indicators currently used for behavioural surveillance.

The questionnaires were sent by email to the person responsible for national HIV surveillance in that country with the option of consulting other colleagues with specialist knowledge to help them complete the questionnaires. The key contact in each country then returned the completed questionnaires, and these were loaded into a password-protected database. The data for each population were analysed separately by an expert member of the project team (see list at the end of the article).

\section{Results}

\section{Behavioural surveillance}

Thirty-one countries were invited to take part in the survey and 27 returned a questionnaire on MSM. Of these 27 countries, 14 reported that there was a system of behavioural surveillance among the MSM population in their country: Belgium, Denmark, Estonia, France, Germany, Ireland, Lithuania, the Netherlands, Norway, Slovenia, Spain, Sweden, Switzerland and the United Kingdom (Table 1).

An additional four countries did not consider that they had such a system but nonetheless had conducted behavioural surveys among

T A B L E 1

HIV and STI behavioural surveillance among men who have sex with men (MSM) in 31 European countries

\begin{tabular}{|c|c|c|c|c|c|c|c|c|c|c|c|c|}
\hline \multirow[b]{2}{*}{ Country } & \multirow{2}{*}{$\begin{array}{l}\text { BS* among } \\
\text { MSM }\end{array}$} & \multicolumn{3}{|c|}{ Year of survey } & \multirow{2}{*}{$\begin{array}{c}\text { Frequency } \\
\text { of surveys } \\
\text { (years) }\end{array}$} & \multicolumn{3}{|c|}{ Sampling and recruitment } & \multicolumn{2}{|c|}{ Data collection } & \multirow[b]{2}{*}{ Coverage } & \multirow{2}{*}{$\begin{array}{c}\text { Sample } \\
\text { size each } \\
\text { year }\end{array}$} \\
\hline & & $\begin{array}{l}\text { First } \\
\text { survey }\end{array}$ & $\begin{array}{c}\text { Last } \\
\text { survey * * }^{*}\end{array}$ & Next survey & & Internet & Gay venues & $\begin{array}{c}\text { Gay } \\
\text { press }\end{array}$ & Internet & $\begin{array}{l}\text { Pen \& } \\
\text { paper }\end{array}$ & & \\
\hline Austria & $\mathrm{N}$ & - & - & - & - & - & - & - & - & - & - & - \\
\hline Belgium & Y & 1992 & 2005 & ? & Irregular & Y & Y & Y & Y & Y & Regional & $500-1100$ \\
\hline Bulgaria $^{+}$ & - & - & - & - & - & - & - & - & - & - & - & - \\
\hline Cyprus & $\mathrm{N}$ & - & - & - & - & - & - & - & - & - & - & - \\
\hline Czech Republic & $\mathrm{N}$ & - & - & - & - & - & - & - & - & - & - & - \\
\hline Denmark & $Y$ & 2000 & 2006 & 2009 & $1-4$ & $Y$ & Y & - & Y & Y & National & 2000 \\
\hline Estonia & Y & 2004 & 2007 & $?$ & 3 & $Y$ & Y & - & $Y$ & $Y$ & National & 300 \\
\hline Finland $^{\star \star \star}$ & $\mathrm{N}$ & 2006 & 2006 & $?$ & NA & - & - & $Y$ & - & $Y$ & National & 400 \\
\hline France & Y & 1985 & 2007 & 2009 & $2-3$ & Y & Y & Y & Y & Y & National & $\begin{array}{l}3000- \\
15000\end{array}$ \\
\hline Germany & Y & 1987 & 2007 & $?$ & $2-3$ & $Y$ & Y & $Y$ & $Y$ & $Y$ & National & $200-6000$ \\
\hline Greece $^{\star \star \star}$ & $\mathrm{N}$ & 2007 & 2007 & $?$ & NA & Y & - & - & Y & - & National & 200 \\
\hline Hungary & $\mathrm{N}$ & - & - & - & - & - & - & - & - & - & - & - \\
\hline Iceland & $\mathrm{N}$ & - & - & - & - & - & - & - & - & - & - & - \\
\hline Ireland & $Y$ & 2000 & 2008 & 2009 & Annually & Y & Y & - & Y & Y & National & $900-1300$ \\
\hline Italy $^{+}$ & - & - & - & - & - & - & - & - & - & - & - & - \\
\hline Latvia $^{\star \star \star}$ & $\mathrm{N}$ & 2001 & 2001 & $?$ & NA & - & Y & - & - & Y & National & 100 \\
\hline Lichtenstein & $\mathrm{N}$ & - & - & - & - & - & - & - & - & - & - & - \\
\hline Lithuania & Y & 2003 & 2007 & $?$ & $1-3$ & $Y$ & Y & - & Y & Y & Regional & $100-200$ \\
\hline Luxembourg & $\mathrm{N}$ & - & - & - & - & - & - & - & - & - & - & - \\
\hline Malta & $\mathrm{N}$ & - & - & - & - & - & - & - & - & - & - & - \\
\hline Netherlands & Y & 2000 & 2008 & 2009 & $1-3$ & Y & Y & - & Y & Y & National & $800-4500$ \\
\hline Norway & Y & 2007 & 2007 & 2009 & 2 & Y & - & - & Y & - & National & 2300 \\
\hline Poland ${ }^{* \star *}$ & $\mathrm{~N}$ & 2004 & 2004 & $?$ & NA & - & $Y$ & - & - & $Y$ & National & $?$ \\
\hline Portugal $^{+}$ & - & - & - & - & - & - & - & - & - & - & - & - \\
\hline Romania $^{+}$ & - & - & - & - & - & - & - & - & - & - & - & - \\
\hline Slovakia & $\mathrm{N}$ & - & - & - & - & - & - & - & - & - & - & - \\
\hline Slovenia & Y & 2000 & 2007 & 2009 & Annually & - & Y & - & - & Y & Regional & 100 \\
\hline Spain & Y & 1995 & 2004 & $?$ & $2-3$ & $Y$ & $Y$ & - & $Y$ & $Y$ & National & $95-900$ \\
\hline Sweden & Y & 2006 & 2008 & 2012 & $2-4$ & $Y$ & - & - & Y & - & National & 3000 \\
\hline Switzerland & Y & 1987 & 2008 & 2009 & $2-3$ & $Y$ & Y & Y & Y & Y & National & $500-1244$ \\
\hline UK & Y & 1993 & 2008 & 2010 & $1-3$ & Y & Y & - & Y & Y & National & $\begin{array}{c}800- \\
15000 \\
\end{array}$ \\
\hline
\end{tabular}

*BS: Behavioural surveillance

** As of September 2008

*** Four countries stated they did not have a system of behavioural surveillance among MSM but nonetheless had conducted behavioural surveys in this subpopulation

+ Four countries did not return a questionnaire concerning MSM (Bulgaria, Italy, Portugal, Romania) 
MSM (Finland, Greece, Latvia and Poland). Latvia has conducted repeat surveys among MSM while Finland, Greece and Poland have only conducted one survey to date.

For simplicity these 18 countries are all described as conducting behavioural surveillance since 15 countries have done repeat surveys, while Finland, Greece and Poland have done only one but may do another one in the future.

The remaining nine countries reported that there was no system of behavioural surveillance among MSM in their country, nor had they conducted behavioural surveys among this population: Austria, Cyprus, Czech Republic, Hungary, Iceland, Liechtenstein, Luxembourg, Malta and Slovakia. No information was available for Bulgaria, Italy, Portugal and Romania since they did not return a questionnaire for MSM (Table 1).

In general, Western European countries established behavioural surveillance among MSM before Central or Eastern European countries. France was the first country to introduce behavioural surveillance among MSM in 1985, followed by Switzerland and
Germany (1987), Belgium (1992), United Kingdom (1993) and Spain (1995). Other Western European countries started later: Denmark, Ireland and the Netherlands in 2000, followed by Finland, Sweden (2006), Norway and Greece (2007). Behavioural surveillance among MSM in Central and Eastern Europe was established from the mid 1990s. The first Central/Eastern European country to conduct a behavioural survey among MSM was Latvia (1997), followed by Slovenia (2000), Lithuania (2003), Poland and Estonia (2004) (Table 1).

\section{Sampling and recruitment}

All countries used convenience samples for behavioural surveillance among MSM. Fourteen of the 18 countries recruited MSM from community venues such as gay bars, clubs and saunas, or had done so in the past (Table 1). In the United Kingdom, men were also recruited through gyms. Five countries used the gay press for recruitment (Belgium, Finland, France, Germany and Switzerland).

Fourteen of the 18 countries recruited men through the Internet, reflecting increased use of the Internet by MSM for meeting sexual

TA B L E 2

Topics most frequently covered in behavioural surveillance among men who have sex with men (MSM) in European countries

\begin{tabular}{|c|c|}
\hline & Number of countries where this topic is covered ${ }^{*}$ \\
\hline \multicolumn{2}{|l|}{ Knowledge and attitudes } \\
\hline Knowledge about HIV/AIDS infection and/or treatments & 14 \\
\hline Knowledge of post-exposure prophylaxis (PEP) & 10 \\
\hline Awareness of prevention activities & 14 \\
\hline \multicolumn{2}{|l|}{ Sexual relationships and sexual partners } \\
\hline Types of partners/relationships, such as regular partner, casual partners & 15 \\
\hline \multicolumn{2}{|l|}{ Sexual activity and lifestyle } \\
\hline Sexual activity, such as number of partners, frequency of sexual contacts & 16 \\
\hline Sexual orientation & 16 \\
\hline Sexual practices & 15 \\
\hline How and where partners are met & 12 \\
\hline \multicolumn{2}{|l|}{ Exposure to risk of infection } \\
\hline Condom use at last intercourse & 14 \\
\hline Condom use with different types of partners & 16 \\
\hline Condom use in different types of sexual practice (e.g. vaginal, anal, oral sex) & 12 \\
\hline Risk reduction strategies (such as negotiated safety, serosorting, positioning) & 11 \\
\hline \multicolumn{2}{|l|}{ HIV and other STI } \\
\hline HIV testing & 15 \\
\hline Current or past STI other than HIV and hepatitis & 14 \\
\hline Result of last HIV test (self-reported) & 15 \\
\hline Result of last HIV test (measured) & 7 \\
\hline \multicolumn{2}{|l|}{ Drugs and substance use } \\
\hline Types of drugs consumed & 12 \\
\hline Use of psycho-active substances (including alcohol) and intercourse & 10 \\
\hline Injecting drug use & 7 \\
\hline \multicolumn{2}{|l|}{ Socio-demographic characteristics } \\
\hline Education & 15 \\
\hline Employment & 12 \\
\hline Nationality and/or ethnic origin & 10 \\
\hline
\end{tabular}

*Information on topics covered was provided by 16 countries. 
partners [3] (Table 1). Only Finland, Latvia, Poland and Slovenia have not yet recruited MSM through the Internet. The first country to use the Internet for recruiting MSM was Belgium in 1998.

Respondent driven sampling (RDS) has been used in two countries only (Estonia and Greece). In Estonia this approach was reportedly not successful. Only 60 men were recruited using this method.

In most countries ( $n=15)$, national samples were recruited although "local" samples were often included too. In the other three countries (Belgium, Lithuania, Slovenia) only local samples were recruited. The local samples were often recruited in cities with large MSM populations such as London, Paris, Geneva or Ljubljana.

\section{T A B L E}

Specific indicators currently used for behavioural surveillance among men who have sex with men (MSM) in European countries

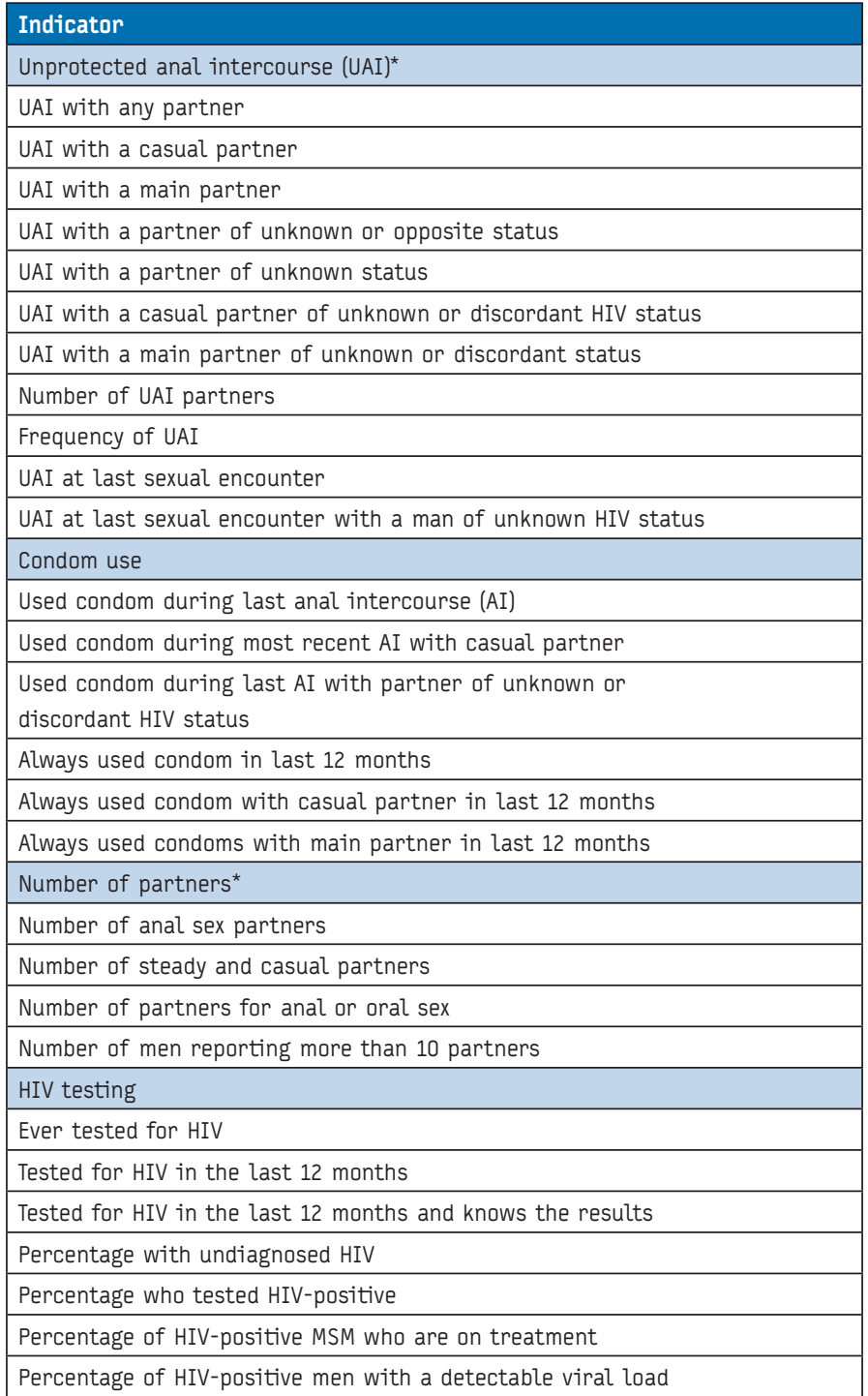

* For UAI and the number of sexual partners, most countries used a 12-month time period but some used a three- or six-month period
Local samples can act as sentinel populations for monitoring time trends in sexual behaviour in metropolitan areas.

Sample size varied between countries. This is not surprising, since the size of the general population, and therefore the MSM population, also varies between countries. But an additional factor was the sampling method. Samples recruited through the Internet were generally larger than those recruited in the community (e.g. in bars, clubs and other venues). For community samples, sample size ranged from 100 (Slovenia, Latvia, Lithuania) to 2,000 (London), while for Internet samples it ranged from 900 (Ireland, Spain) to 15,000 (France).

\section{Data collection}

Surveys conducted in community venues (e.g. bars, clubs, gyms, saunas) or through the gay press used pen-and paper questionnaires for data collection ( $n=15$ countries), but increasing use of the Internet for recruitment has led to the use of web surveys completed online. In the last five years, 14 countries have conducted behavioural surveillance among MSM using web surveys (Table 1). Of these 14 countries, three (Greece, Norway, Sweden) used exclusively online surveys for data collection while 11 also used pen-and-paper questionnaires for their community samples. Greece, Norway and Sweden all introduced behavioural surveillance after 2000

Thirteen countries reported collecting behavioural surveillance data regularly, while five did not (Belgium, Finland, Greece, Latvia, Poland). In those five countries, the cross-sectional surveys were conducted on an ad hoc basis.

In the thirteen countries that conducted surveys regularly, the interval between surveys varied widely. Some countries (e.g. United Kingdom) conducted cross-sectional surveys annually among MSM while others conducted their surveys every 3-5 years.

Nine countries reported they would repeat the survey between 2009 and 2012. In the remaining nine countries, a decision has yet to be made about future surveys.

\section{Topics currently covered}

Sixteen of the 18 countries provided information on the range of topics covered in their behavioural surveys. The topics most commonly covered among MSM are presented in Table 2. Most of these topics were surveyed regularly as part of a country's behavioural surveillance programme. The exceptions were 'Risk reduction strategies' and 'How and where men met their sexual partners'. About half the countries covered these regularly, while the other half did so irregularly.

\section{Current behavioural surveillance indicators}

Ten of the 18 countries provided information on their current behavioural surveillance indicators, (Belgium, Denmark, France, Lithuania, the Netherlands, Slovenia, Spain, Sweden, Switzerland, the United Kingdom).

Four main indicators are in current use in most of these countries. These are:

- Unprotected anal intercourse

- Condom use

- Number of partners

- HIV testing 
However, there was considerable diversity between countries in the specific indicators as can be seen in Table 3 .

\section{Discussion}

This study, mapping HIV and STI behavioural surveillance in Europe, identified 14 countries with a system of behavioural surveillance among MSM and another four that had conducted behavioural surveys in this population. Nine countries did not have a system of behavioural surveillance among MSM and a further four countries did not provide any information.

The study revealed considerable diversity between countries in when behavioural surveillance began, the range of indicators used and how frequently surveys are repeated. For example, Western European countries generally introduced behavioural surveillance among MSM before Central or Eastern Europe, but this was not always the case. On the other hand, since there is no sampling frame for MSM, all European countries have used "convenience samples" for behavioural surveillance in this population, often recruited through the Internet.

\section{Indicators}

Although a wide range of behavioural surveillance indicators is currently used, these can be grouped under four main headings: unprotected anal intercourse, condom use, number of partners and HIV testing. A consensus appears to have emerged across Europe as to which are the most important indicators for behavioural surveillance among MSM [4,5]. However, there is also enormous variation between countries in the specific indicators used which makes direct comparison between countries problematic.

One way forward would be for all countries to incorporate a core set of indicators for behavioural surveillance among MSM. The suggested set of indicators is summarised below.

Suggested set of indicators for behavioural surveillance among $\underline{\mathrm{MSM}}$

- Unprotected anal intercourse (UAI) with a partner of unknown or discordant HIV status in the last 12 months (overall and separately for casual and main partners)

- Unprotected anal intercourse (UAI) with a casual partner of the same HIV status in the last 12 months

- Diagnosed with an STI in the last 12 months

- Tested for HIV in last 12 months

- Percentage who are HIV-positive

- Number of sexual partners in last 6 or 12 months (male and female)

- Used condom at last anal intercourse (distinguishing between casual and main partners)

- Where men met their sexual partners in the last 12 months (saunas, bars, clubs, Internet, etc.)

The suggested UAI indicators need to differentiate: (i) between main and casual partners and (ii) between a partner of unknown or discordant HIV status and a partner of the same HIV status ("serosorting").

\section{Main and casual partners}

It is important to differentiate between main and casual UAI partners of unknown or discordant HIV status. This differentiation is important since, in some European countries, HIV transmission among MSM is more likely to occur within a regular relationship [6] while in other countries it is more likely to occur with a casua partner [7]. Collecting data on UAI with main as well as casual partners of unknown or discordant HIV status, will allow us to identify the context in which HIV transmission occurs among MSM in Europe.

\section{Partners of the same HIV status}

An increasing number of HIV-positive men report 'serosorting' with casual partners, i.e. only having UAI with casual partners who are also HIV-positive [8,9]. In principle, this does not present a risk of HIV transmission to someone who is uninfected, but it does present a risk for STI transmission among HIV-positive MSM $[10,11]$. Serosorting has undoubtedly contributed to the recent increase in STI among HIV-positive MSM in Western Europe and therefore needs to be monitored [12]. In addition, some HIVnegative men report serosorting with casual partners as a risk reduction strategy $[13,14]$. Since it is extremely difficult for HIVnegative men to establish seroconcordance reliably in a casual encounter, serosorting with casual partners among HIV-negative men presents a risk for HIV transmission.

The suggested set of indicators could provide a template for behavioural surveillance in European countries. The suggested indicators incorporate those recommended by the United Nations General Assembly Special Session (UNGASS) but in some respects they are more precise. For example, our suggested set of indicators differentiates between main and casual partners.

The Joint United Nations Programme on HIV/AIDS (UNAIDS) and the World Health Organization (WHO) recommend that behavioural surveillance and biological surveillance be combined as part of a second generation surveillance system. In this way, behavioural and biological data can be used to validate one another. "Two sets of data pointing in the same direction make a more convincing case than just behavioural data or HIV prevalence alone" [2].

\section{Sampling}

One of the challenges for conducting behavioural surveillance among MSM is that there is no sampling frame from which to draw a probability sample [15]. To date, it has been impossible to assess the size of the MSM population in any one country since questions on sexual identity or sexual behaviour are not routinely included in national censuses. Questions on sexual behaviour or identity may be included in some national probability surveys, but these studies usually recruit relatively small numbers of MSM [16]. As a consequence, behavioural surveillance among MSM in all European countries relies on 'convenience samples'.

In Europe 18 countries have conducted cross-sectional surveys in convenience samples of MSM recruited through a number of channels including the gay press, bars and gyms. An increasing number of countries now recruit men through the Internet. In the last five years, fourteen of the eighteen countries conducting behavioural surveillance or surveys among MSM have used the Internet for recruitment and data collection. This reflects the well established trend for MSM to meet sexual partners through the Internet via dating sites [17-19]. Three of the four countries that have not yet used the Internet for behavioural surveillance were in Central or Eastern Europe (Latvia, Poland, Slovenia).

An advantage of using convenience samples is that it is possible to recruit large numbers of MSM who may be at risk for HIV and STI, at relatively low cost. This is especially true for Internet samples. On the other hand, the disadvantage is that convenience samples are not representative of the overall MSM population [15]. 
While it is possible to recruit quasi-probability samples of MSM using time-location sampling in venues $[20,21]$, this can be costly when compared with recruiting men through the Internet. Since behavioural surveillance requires cross-sectional surveys to be repeated at regular intervals, keeping the cost down is a priority for many countries. In general, convenience samples, including those recruited through the Internet, tend to overestimate the true level of HIV or STI risk in the MSM population [22,23]. However, if sampling bias remains constant from one cross-sectional survey to the next, then for surveillance purposes it is possible to monitor time trends in risk behaviour using convenience samples with some degree of reliability.

Two countries used respondent driven sampling (RDS) to recruit MSM for behavioural surveillance. In Estonia only 60 men were recruited using RDS; they had hoped to recruit 400 men using this method. It seems that RDS reached a less diverse group of MSM in Estonia than recruitment through the Internet. There was a number of reasons why RDS did not work in Estonia [24]. Because employment rates are high in Estonia, there was relatively little interest in the financial reward for taking part in the RDS survey. Also, the opportunity to have a free HIV/STI test was not hugely attractive in a country where testing is widely available. In addition, married men or men with girlfriends were afraid of taking part in an RDS survey for MSM, whereas they were more willing to take part in an Internet survey. The Estonian experience highlights the importance of examining context when using RDS for recruiting MSM for behavioural surveillance [24].

\section{Gaps, opportunities and limitations}

Several important gaps have emerged from this mapping exercise. The most striking is that nine European countries have not introduced behavioural surveillance among MSM (and another four did not provide any information) even though MSM remain the group most at risk of acquiring HIV in many European countries [1]. Some of these countries have too small a population to justify conducting behavioural surveillance among MSM. It is important to recognise that in some European countries MSM may be harder to reach than in others because of cultural or religious barriers.

Behavioural surveillance among gay men provides an opportunity to collect detailed information about the behaviour of HIV-positive gay men (as well as those who are HIV negative or untested). Very few European countries have established a programme of behavioural surveillance among people living with HIV. Consequently, behavioural surveys in population groups where the prevalence of HIV is relatively high (e.g. gay men) allows trends in behaviour to be monitored specifically among gay men living with HIV.

Although the majority of European countries now recruit gay men online for behavioural surveillance (i.e. via the Internet), little is known about the websites used in those countries. Do different websites attract different kinds of men? If so, selection bias could vary from one country to another which could affect comparisons of behavioural data between countries.

A limitation of this analysis is that it depends on the accuracy and completeness of the data provided by Member States concerning behavioural surveillance in each country. Nonetheless, as can be seen in the full report (see table 8.3 p. 90-6) those countries that returned a questionnaire provided comprehensive and detailed information. An additional point is that four countries did not return the MSM questionnaire although a review of the literature suggests that behavioural research has been conducted in some of those countries [4].

\section{Conclusion}

In conclusion, this study mapping behavioural surveillance among MSM in Europe has revealed both similarities and differences between countries. In a number of European countries behavioural surveillance among MSM has developed along similar lines without formal coordination. On the other hand, the diversity of behavioural indicators limits the extent to which direct comparisons can be made between countries. As part of its mandate to coordinate surveillance of communicable disease in the EU, the European Centre for Disease Prevention and Control (ECDC) will support and encourage harmonisation of behavioural surveillance among MSM in EU Member States.

\section{Acknowledgements}

The organisers of the project would like to thank all participating countries for completing the questionnaires and providing relevant material for the original report. J Elford would like to thank Edith Stokes and the staff at Mount Pleasant where this paper was first drafted.

The project was funded by the European Centre for Disease Prevention and Control (ECDC), contract No ECD 903

The HIV and STI Behavioural Surveillance Mapping Group:

The full report (ECDC Technical Report. Mapping of HIV/STI behavioural surveillance in Europe) was commissioned by the European Centre for Disease Prevention and Control (ECDC), coordinated by Marita van de Laar and produced by the Institute for Social and Preventive Medicine (IUMSP), University of Lausanne, Switzerland working with an international team of experts listed below. Each expert focused on one population group (in brackets).

- $\quad$ Françoise Dubois-Arber, Institute for Social and Preventive Medicine (IUMSP), Lausanne, Switzerland (team leader, youth);

- $\quad$ Brenda Spencer, IUMSP. Lausanne, Switzerland (general population);

- Vivian Hope, London School of Hygiene and Tropical Medicine, United Kingdom (IDU);

- Jonathan Elford, City University, London, United Kingdom (MSM);

- $\quad$ France Lert, Institut national de la santé et de la recherché médicale, France (PLWHA);

- $\quad$ Helen Ward, Imperial College, London, United Kingdom (sex workers);

- $\quad$ Nicola Low, Institute for Social and Preventive Medicine, Bern, Switzerland (STI clinic patients);

- Mary Haour-Knipe, freelance consultant (formerly IOM) (migrants and ethnic minorities);

- $\quad$ André Jeannin, IUMSP, Lausanne, Switzerland (organisation of survey);

- Jean-Pierre Gervasoni, IUMSP, Lausanne, Switzerland (organisation of survey)

- Marie-Jeanne Pellaz, IUMSP, Lausanne, Switzerland (secretarial assistance);

- $\quad$ Bertrand Graz, IUMSP, Lausanne, Switzerland (literature review);

- Marita van de Laar, ECDC, Stockholm, Sweden (coordinator).

The full report is available at:

http://www.ecdc.europa.eu/en/publications/Publications/0909_TER_Mapping_of_HIV_ STI_Behavioural_Surveillance_in_Europe.pdf

\section{References}

1. European Centre for Disease Prevention and Control / WHO Regional Office for Europe: HIV/AIDS Surveillance in Europe 2007. Stockholm: European Centre for Disease Prevention and Control; 2008. Available from: http://ecdc.europa.eu/ en/publications/Publications/0812_SUR_HIV_AIDS_surveillance_in_Europe.pdf

2. World Health Organization (WHO) and Joint United Nations Programme on HIV/AIDS (UNAIDS). Guidelines for second generation surveillance. Geneva: WHO; 2000. Available from: http://www.emro.who.int/gfatm/guide/tools/ unaidssurveillance/unaidssurveillance.pdf

3. Elford J. Changing patterns of sexual behaviour in the era of highly active antiretroviral therapy. Curr Opin Infect Dis. 2006;19(1):26-32. 
4. Bochow M, Chiarotti F, Davies P, Dubois-Arber F, Dür W, Fouchard J, et al. Sexual behaviour of gay and bisexual men in eight European countries. AIDS Care. 1994;6(5):533-49.

5. Hubert M. Studying and comparing sexual behaviour and HIV/AIDS in Europe. In: Hubert M, Bajos N, Sandfort TGM. Editors. Sexual behaviour and HIV/AIDS in Europe. London: UCL Press; 1998.

6. Davidovich U, de Wit J, Albrecht N, Geskus R, Stroebe W, Coutinho R. Increase in the share of steady partners as a source of HIV infection: a 17-year study of seroconversion among gay men. AIDS. 2001;15(10):1303-8.

7. Elford J, Bolding G, Davis M, Sherr L, Hart G. Trends in sexual behaviour among London gay men 1998-2003: implications for STI/HIV prevention and sexual health promotion. Sex Transm Infect. 2004;80(6):451-4.

8. Halkitis PN, Green KA, Remien RH, Stirratt MJ, Hoff CC, Wolitski RJ, Parsons JT. Seroconcordant sexual partnerings of HIV-seropositive men who have sex with men. AIDS. 2005;19 Suppl 1:S77-86.

9. Parsons J, Schrimshaw E, Wolitski R, Halkitis P, Purcell D, Hoff C, et al. Sexual harm reduction practices of HIV-seropositive gay and bisexual men: serosorting, strategic positioning and withdrawal before ejaculation. AIDS. 2005 Apr;19 Suppl 1:S13-25.

10. Gotz H, van Doornum G, Niesters H, den Hollander J, Thio H, de Zwart O. A cluster of acute hepatitis $C$ virus infection among men who have sex with men - results from contact tracing and public health implications. AIDS 2005; 19(9):969-74.

11. Urbanus AT, van de Laar TJ, Stolte IG, Schinkel J, Heijman T, Coutinho RA, et al. Hepatitis $C$ virus infections among HIV-infected men who have sex with men: an expanding epidemic. AIDS. 2009;23(12):F1-7.

12. Dougan S, Evans BG, Elford J. Sexually transmitted infections in Western Europe among HIV-positive men who have sex with men. Sex Transm Dis. 2007;34(10):783-90.

13. Mao L, Crawford J, Hospers H, Prestage G, Grulich A, Kaldor J, et al. "Serosorting" in casual anal sex of HIV-negative gay men is noteworthy and is increasing in Sydney, Australia. AIDS 2006;20(8):1204-6.

14. Elford J, Bolding G, Sherr L, Hart G. No evidence of an increase in serosorting with casual partners among HIV-negative gay men in London, 1998-2005. AIDS. 2007;21(2):243-5.

15. McGarrigle C, Fenton K, Gill O, Hughes G, Morgan D, Evans B. Behavioural surveillance: the value of national coordination. Sex Transm Infect. 2002;78(6):398-405.

16. Mercer C, Fenton KA, Copas A, Wellings K, Erens B, McManus S, et al. Increasing prevalence of male homosexual partnerships and practices in Britain 19902000; evidence from national probability surveys. AIDS. 2004;18(10):1453-8.

17. Hospers H, Kok G, Harterink P, de Zwart O. A new meeting place: chatting on the Internet, e-dating and sexual risk behaviour among Dutch men who have sex with men. AIDS. 2005 Jul 1;19(10):1097-101.

18. Bolding G, Davis M, Hart G, Sherr L, Elford J. Gay men who look for sex on the Internet: is there more HIV/STI risk with online partners? AIDS. 2005;19(9):961-8.

19. McFarlane M, Bull SS, Rietmeijer CA. The Internet as a newly emerging risk environment for sexually transmitted diseases. JAMA. 2000;284(4):443-6.

20. Mackellar DA, Gallagher KM, Finlayson T, Sanchez T, Lansky A, Sullivan PS. Surveillance of HIV risk and prevention behaviors of men who have sex with men--a national application of venue-based, time-space sampling. Public Health Rep. 2007;122 Suppl 1:39-47.

21. Gallagher KM, Sullivan PS, Lansky A, Onorato IM. Behavioral surveillance among people at risk for HIV infection in the U.S.: the National HIV Behavioral Surveillance Public Health Rep. 2007;122 Suppl 1:32-8.

22. Dodds JP, Mercer CH, Mercey DE, Copas AJ, Johnson AM. Men who have sex with men: a comparison of a probability sample survey and a community based study. Sex Transm Infect. 2006;82(1):86-7.

23. Evans AR, Wiggins RD, Mercer CH, Bolding GJ, Elford J. Men who have sex with men in Great Britain: comparison of a self-selected internet sample with a national probability sample. Sex Transm Infect. 2007;83(3):200-5.

24. Johnston LG, Trummal A, Lohmus L, Ravalepik A. Efficacy of convenience sampling through the Internet versus respondent driven sampling among males who have sex with males in Tallinn and Harju County, Estonia: challenges reaching a hidden population. AIDS Care. 2009;21:1195-202.

25. Malekinejad M, Johnston LG, Kendall C, Kerr LR, Rifkin MR, Rutherford GW. Using respondent-driven sampling methodology for HIV biological and behavioral surveillance in international settings: a systematic review. AIDS Behav. 2008;12(4 Suppl):S105-30. 